\title{
Retrospective Development of a Novel Resilience Scale using Existing Cohort Data: The Adolescent to Adult Health Resilience Instrument
}

Diana Montoya-Williams ( $\square$ montoyawid@email.chop.edu )

https://orcid.org/0000-0002-5882-1181

Molly Passarella

Children's Hospital of Philadelphia

Scott A Lorch

Children's Hospital of Philadelphia

Research article

Keywords: resilience, validation, Add Health, scale development

Posted Date: August 15th, 2019

DOl: https://doi.org/10.21203/rs.2.12964/v1

License: (c) (i) This work is licensed under a Creative Commons Attribution 4.0 International License.

Read Full License 


\section{Abstract}

Background: Cohort studies represent rich sources of data that can be used to link components of resilience to a variety of health-related outcomes. The Adolescent to Adult Health (Add Health) cohort study represents one of the largest data sets of the health and social context of adolescents transitioning into adulthood. It did not however use validated resilience scales in its data collection process. This study aimed to retrospectively create and validate a resilience scale using existing data from the cohort to better understand the resilience of its participants. Methods: Questions asked of participants during one Add Health data collection time period ( $\mathrm{N}=9852)$ were matched to items on a well-known and widely validated resilience scale called the Connor Davidson Resilience Scale. Factor analysis and psychometric analyses were used to refine and validate this novel Adolescent to Adult Health Resilience Instrument. Construct validity utilized participants' answers to the 10 item Center for Epidemiologic Studies Depression Scale, which has been used to validate other resilience scales. Results: Factor analysis yielded an instrument with 13 items that showed appropriate internal consistency statistics. Resilience scores in our study were normally distributed with no ceiling or floor effects. Our instrument had appropriate construct validity, negatively correlating to answers on the depression scale $(r=-0.64$, $p<0.001)$. We also found demographic differences in mean resilience scores: lower resilience scores were seen among women and younger participants. Resilience scores increased with increasing education and household income. Conclusions: It is possible to retrospectively construct a resilience scale from existing cohort data and achieve good psychometric properties. The Adolescent to Adult Health Resilience Instrument can be used to better understand the relationship between resilience, social determinants of health and health outcomes among young adults using existing data, much of which is publicly available. Keywords: resilience, validation, Add Health, scale development

\section{Background}

Resilience is defined as an individual's ability to positively adapt in the face of stress and/or adversity so as to maintain in relatively stable or even good psychological and physical health. ${ }^{1,2}$ Research on resilience and health has increased substantially in recent years due to a shift in focus from studying health predominantly from a pathogenic orientation (i.e. what causes disease) to exploring salutogenic forces (i.e what promotes health). ${ }^{3}$ Individual psychological resilience appears to be protective against a variety of physical and mental health outcomes such as depression, anxiety and early mortality. ${ }^{4,5}$

Most studies looking at resilience and its relationship to health and disease have prospectively evaluated individual resilience through the use of resilience scales. ${ }^{4}$ Although a wide range of scales exist, most of them are multi-dimensional, measuring several constructs or themes. ${ }^{4,6}$ Some of the more commonly recurrent constructs that are believed to represent high resilience and thus are often included in validated scales are a sense of personal agency, adaptive coping style, optimism and hopefulness and social support. $4,7,8$ However, given the costly and time-consuming nature of conducting prospective studies to assess resilience in a population using a validated scale, some groups have utilized alternate strategies 
to assess individual resilience in a retrospective fashion, using existing data from large cohorts. An example of one large nationally representative cohort whose data has been utilized in this way is the Adolescent to Adult Health (Add Health) cohort study. The Add Health cohort study is a nationallyrepresentative longitudinal cohort study that recruited students in the United States in grades 7-12 during the 1994-95 school year. After following adolescents into adulthood with in-home surveys collected over five waves of data collection spanning 24 years, it represents one of the broadest data sets on the social, economic, academic, psychological and physical health status of adolescents transitioning into adulthood. ${ }^{9}$

Add Health explores both risk and protective factors for young adult health and achievement. Many of the questions asked of participants over the years relate to themes of self-efficacy, optimism, persistence, social support and faith/religiosity--themes that make up many validated resilience scales. However validated resilience scales were not used during any of the interviews. Researchers interested in the relationship between these themes and health have had to use alternate strategies to study resilience in this cohort, often focusing on single discrete concepts such as agency, self-efficacy or optimism. ${ }^{8,10,11}$ We built on this type of work by aiming to create an Add Health-based global resilience instrument that mirrored the structure of an existing widely used and well-validated resilience scale. The creation of such an instrument would allow the Add Health cohort study dataset to be used to more broadly examine resilience and its relationship to health outcomes, health behaviors and social contexts.

\section{Methods}

\section{Participants \& Data Source}

The present study uses data specifically from the fourth wave of data collection from Add Health participants. Conducted in 2008, this wave consisted of in-home interviews of 15,701 young adults ages 24-32. We excluded participants who had not answered the questions that became candidate items for our Add Health Resilience Instrument, which left 9852 participants in this validation study population.

Although some Add Health data is publicly available via the Add Health website (https://www.cpc.unc.edu/projects/addhealth/documentation/publicdata), this study utilized the extensive restricted-use data available by contractual agreement. This study was deemed exempt by our local institutional review board to the publicly available de-identified nature of the Add Health data.

\section{Model Resilience Scale}

A recent systematic review of resilience measurement scales by Windle et al. found that there is no current gold standard amongst the scales that have published psychometric validation data, in part because there is no gold standard for criterion validity for resilience. ${ }^{6}$

Windle's group did conclude that the Connor-Davidson Resilience (CD-RISC) Scale, the Resilience Scale for Adults and the Brief Resilience Scale had the best psychometric ratings. ${ }^{6}$ The CD-RISC was initially 
developed as a 25 item scale, ${ }^{12}$ and has been validated in a wide range of subjects of varying ages. ${ }^{13-15}$ More recently, Campbell-Sills and Stein developed a 10 item version of the CD-RISC, ${ }^{16}$ which has also been validated in a variety of cross-cultural populations. ${ }^{17,18}$ Given the widespread use of the CD-RISC scales and the breadth of their existing validation literature, we used the CD-RISC as the model for the Add Health Resilience Instrument.

\section{Items from Add Health}

The original CD-RISC is composed of 5 major domains: personal competence or tenacity, strengthening effects of stress, positive acceptance of change, control and spiritual influences. ${ }^{12}$ In order to capture any Add Health data that might indicate resilience, all Add Health questions that reflected the items on the original expanded CD-RISC were pulled from the Add Health Wave 4 interview dataset. This led to 21 candidate Add Health items that were evaluated for inclusion in our Add Health Health Resilience Instrument (AHRI).

\section{Statistical Analyses}

Principal component analyses of the 21 Add Health items were conducted on all available responses using oblique rotation to allow for inter-item correlation. Eigenvalues $>1$ were retained. Internal consistency was evaluated by using Cronbach's alpha, where the recommended value ranges from 0.7 to 0.95. ${ }^{19}$ We also assessed for internal consistency using item-test, item-rest and inter-item correlations. Item-test correlations determine how well each item correlates with the overall scale and should be roughly similar for all items. ${ }^{20}$ The item-total correlation shows how the item correlates with a scale computed from only the other items; ideal values are above $0.2 .{ }^{21}$ Inter-item correlations identify items too similar or not similar enough in a scale, with recommended values between 0.2 and $0.5 .{ }^{22}$

After arriving at a scale where included items showed the most optimal internal consistency, items were reverse coded if negatively worded in order for higher scores to indicate higher resilience. Responses were coded into a likert type scale, with the highest score indicating a participant "strongly agreed" with a positive statement. There was no differential weighting for items; questions which asked participants to pick the frequency of feeling certain positive attributes over the past month had a maximum score of 3 while all other items had a maximum score of 2 . This was done to better reflect the original potential answer choices as defined by Add Health investigators. Scores were them summed to create an overall AHRI score. Descriptive statistics were used to characterize the AHRI scores first in the overall population, then by gender and age. Ceiling and floor effects were analyzed by calculating the frequency of participants showing the minimum and maximum possible scores. Floor and ceiling effects of greater than $15 \%$ indicates limited content validity.(Terwee et al. 2007)

As there are no gold standard criterion validity measures for resilience, we evaluated the AHRI through construct validity as others have done for the validation of other resilience scales. ${ }^{16,23}$ High levels of resilience are known to be protective against adverse mental health outcomes like depression 24,25 or post- 
traumatic stress disorder. ${ }^{26}$ Thus, we evaluated for discriminant validity by calculating the correlation between AHRI scores and participants' scores on a depression scale that had been originally embedded in the fourth wave of Add Health in home interviews. During the original 2008 wave of data collection, participants completed the short form of the Centers for Epidemiologic Studies Depression Scale (CESD10). Initially developed with 20 items, ${ }^{27}$ the CESD-10 scale is widely used for the identification and evaluation of depression in the general and adolescent populations. ${ }^{28,29}$ Various shorter forms of the 20item CESD have been evaluated over the years, including the CESD-10 developed by Andresen et al. ${ }^{30}$ Using the existing CESD-10 data present in the original 2008 Add Health dataset, we were able to define a depression score for participants in our cohort. Possible responses for CESD-10 related items ranged from never/rarely (0) to most/all the time (3) resulting in a possible scale of $0-30$. We categorized a score $>10$ as indicative of adult depressive symptoms as has been recommended and done by others using this data for a similar purpose. ${ }^{31-33}$ Resilience scores calculated using our AHRI were correlated with CESD-10 scores. In addition, we created three levels of Add Health resilience (low, medium or high) and compared CESD-10 scores in each of these three resilience categories via ANOVA analyses. Finally, we assessed for differences in AHRI scores between participants who had ever received a diagnosis of anxiety, depression or post-traumatic stress disorder in their lives and those who had not using Chi Square tests.

Add Health oversampled certain subgroups by design, thus all analyses of this dataset required survey weighting in order for results to remain nationally representative. ${ }^{9}$ The software STATA, version 14 , was used for all statistical analyses.

\section{Results}

\section{Study Population}

Table 1 depicts the demographics of our cohort and overall resilience score distribution. The average age was 28.8 years (SD 1.8) and most participants were female (57\%). The racial/ethnic makeup of the cohort was similar to that reported in the 2008 American Community Survey Data ${ }^{34}$, which helped confirm the national representativeness of our cohort and verified that survey results were weighted appropriately. The majority of participants in our study (69.2\%) finished high school but had not completed college. In addition, $46 \%$ of the cohort had a household income of less than $\$ 50,000$ a year, which was slightly lower than the median household income reported by the Census Bureau's in $2008 .{ }^{34}$ 


\begin{tabular}{lc}
\hline \hline \multicolumn{1}{c}{ Table 1. Demographic Characteristics (N=9852) } \\
\hline \multicolumn{1}{c}{ Variable } & Mean (SD) or \% of Cohort \\
\hline Age (yrs) & $28.8(1.8)$ \\
Gender (\% Female) & $57.0 \%$ \\
\hline Race/Ethnicity & $64.2 \%$ \\
\hline Non-Hispanic White & $15.8 \%$ \\
\hline Non-Hispanic Black & $2.5 \%$ \\
\hline Non-Hispanic American Indian & $2.9 \%$ \\
\hline Non-Hispanic Asian & $0.9 \%$ \\
\hline Other Non-Hispanics & $13.6 \%$ \\
\hline Hispanic & $12.9 \%$ \\
\hline Highest Level of Education Achieved & $69.2 \%$ \\
\hline Less than High School & $11.6 \%$ \\
\hline Less than College & $6.3 \%$ \\
\hline College Degree & \\
\hline More than College & $12.0 \%$ \\
\hline Household Income & $34.2 \%$ \\
\hline$<\$ 20,000$ & $38.1 \%$ \\
\hline \$20-49,999 & $7.6 \%$ \\
\hline $50-99,999$ & $3.0 \%$ \\
\hline \$100-149,999 & $5.0 \%$ \\
\hline Missing 000 & \\
\hline & \\
\hline & \\
\hline & \\
\hline & \\
\hline &
\end{tabular}

\section{Psychometric Analyses}

Principal component analysis was conducted on the 21 candidate Add Health items that aligned with specific CD-RISC items. Items which lowered the scale's overall Cronbach's alpha were eliminated until we arrived at a 13-item AHRI with 3 factors that had an overall alpha of 0.78 . Items that were eliminated were related to being stressed easily, the strength of a partner's commitment, the total number of close friends and participants religiosity (which made up part of the extended 25 item CD RISC but not the refined 10 item version). Cronbach's alpha for each factor ranged from 0.47 to 0.75 . The average inter-item correlation was 0.22 (Table 2). 


\begin{tabular}{|c|c|c|c|c|c|c|c|}
\hline Factor & Item & $\begin{array}{l}\text { Factor } \\
\text { Loading }\end{array}$ & $\begin{array}{l}\text { Loading } \\
\text { Sign }\end{array}$ & $\begin{array}{l}\text { Item-test } \\
\text { correlation }^{\mathrm{a}}\end{array}$ & $\begin{array}{c}\text { Item-total } \\
\text { Correlation }\end{array}$ & $\begin{array}{l}\text { Average inter- } \\
\text { item correlation }\end{array}$ & Alphad $^{\mathrm{d}}$ \\
\hline \multirow{8}{*}{$\begin{array}{c}\text { Factor } \\
1\end{array}$} & & & & & & & 0.7468 \\
\hline & 1 & 0.4452 & + & 0.5924 & 0.4845 & 0.2096 & 0.7609 \\
\hline & 2 & 0.2413 & + & 05532 & 0.4391 & 0.2137 & 0.7653 \\
\hline & 3 & 0.7630 & + & 0.5359 & 0.4191 & 0.2155 & 0.7672 \\
\hline & 4 & 0.4468 & + & 0.5286 & 0.4107 & 0.2162 & 0.7680 \\
\hline & 5 & 0.7306 & + & 0.5792 & 0.4691 & 0.2110 & 0.7624 \\
\hline & 6 & 0.7323 & + & 0.5892 & 0.4807 & 0.2100 & 0.7613 \\
\hline & 7 & 0.5052 & + & 0.4413 & 0.3123 & 0.2253 & 0.7772 \\
\hline
\end{tabular}

\begin{tabular}{cccccccc}
\hline $\begin{array}{c}\text { Factor } \\
2\end{array}$ & & & & & & & $\mathbf{0 . 6 8 6}$ \\
& 8 & 0.7089 & - & 0.5980 & 0.4912 & 0.2090 & 0.7603 \\
& 9 & 0.6918 & - & 0.5009 & 0.3793 & 0.2192 & 0.7711 \\
& 10 & 0.8220 & - & 0.5825 & 0.4730 & 0.2107 & 0.7621 \\
\hline $\begin{array}{c}\text { Factor } \\
\mathbf{3}\end{array}$ & & & & & & & $\mathbf{0 . 4 7 2 7}$ \\
& 11 & 0.4367 & - & 0.4059 & 0.2732 & 0.2289 & 0.7808 \\
& 12 & 0.7001 & - & 0.4973 & 0.3751 & 0.2195 & 0.7714 \\
& 13 & 0.7422 & - & 0.4365 & 0.3071 & 0.2258 & 0.7777 \\
\hline
\end{tabular}

Overall

AHRI

$0.2165 \quad 0.7822$

${ }^{a}$ Ideally these values are similar for all items.

b Recommended values are above 0.2.

${ }^{\mathrm{C}}$ Recommended values are between 0.2 and 0.5 .

d Ideal values are between 0.7 and 0.95 .

We labeled the 3 factors within the AHRI as personal competence, feeling supported vs. overwhelmed, and optimism. Each item loaded predominantly on only one factor (Table 3). The maximum possible score on our AHRI was 29. Resilience scores in our study population using this constructed scale appeared normally distributed (Supplementary Figure 1). The skewness (0.01) and kurtosis (2.8) of the 
resilience scores were also consistent with a normal distribution. There were no ceiling or floor effects in our cohort (Less than $1 \%$ of participants scored either the minimum or maximum) ${ }^{19}$ Mean resilience score for this population was 14.1. Low AHRI scores were defined as $<10$, medium as 10-19 and high as 20-29. The majority of the cohort (68\%) fell into the medium resilience category.

Table 3. Add Health Resilience Instrument

\begin{tabular}{|c|c|c|c|}
\hline Item & $\begin{array}{l}\text { Max } \\
\text { Score }\end{array}$ & $\begin{array}{l}\text { Mean } \\
\text { Score in } \\
\text { Cohort }\end{array}$ & $\begin{array}{l}\text { Mean Factor } \\
\text { Score if } \\
\text { Depressed }\end{array}$ \\
\hline Factor 1. Personal Competence/Agency & 15 & 7.1 & 4.8 \\
\hline $\begin{array}{l}\text { 1. There are many things that interfere with what I } \\
\text { want to do. }\end{array}$ & 2 & 0.6 & \\
\hline $\begin{array}{l}\text { 2. In the last } 30 \text { days, I have often felt confident in } \\
\text { my ability to handle my personal problems. }\end{array}$ & 3 & 2.1 & \\
\hline $\begin{array}{l}\text { 3. There is little I can do to change the important } \\
\text { things in my life. }\end{array}$ & 2 & 1.0 & \\
\hline 4. I hardly ever expect things to go my way. & 2 & 0.6 & \\
\hline $\begin{array}{l}\text { 5. Other people determine most of what I can and } \\
\text { cannot do. }\end{array}$ & 2 & 1.1 & \\
\hline $\begin{array}{l}\text { 6. There is really no way I can solve the problems I } \\
\text { have. }\end{array}$ & 2 & 1.1 & \\
\hline $\begin{array}{l}\text { 7. I go out of my way to avoid having to deal with } \\
\text { problems in my life. }\end{array}$ & 2 & 0.7 & \\
\hline Factor 2: Social Support/ Feeling Overwhelmed & 8 & 4.7 & 2.2 \\
\hline $\begin{array}{l}\text { 8. In the last } 30 \text { days, I have often felt that } \\
\text { difficulties were piling up so high that I could not } \\
\text { overcome them. }\end{array}$ & 3 & 1.8 & \\
\hline 9. I often feel isolated from others. & 2 & 1.2 & \\
\hline $\begin{array}{l}\text { 10. In the last } 30 \text { days, I have often felt unable to } \\
\text { control the important things in my life. }\end{array}$ & 3 & 1.8 & \\
\hline Factor 3. Optimism & 6 & 2.4 & 1.6 \\
\hline 11. I am not easily bothered by things. & 2 & 0.5 & \\
\hline $\begin{array}{l}\text { 12. Overall, I expect more good things to happen to } \\
\text { me than bad. }\end{array}$ & 2 & 1.0 & \\
\hline 13. I'm always optimistic about my future. & 2 & 0.9 & \\
\hline Instrument Totals & 29 & 14.1 & 9.0 \\
\hline
\end{tabular}

In looking at differences in resilience by basic demographics, we found that the mean AHRI score was significantly lower among women compared to men ( 13.8 vs $14.4, p<0.001)$ (Table 4). Participants in their 30 s had slightly higher resilience scores than participants in their $20 \mathrm{~s}(14.5 \mathrm{vs} .13 .9, \mathrm{p}=0.002)$. In addition, resilience scores increased with increasing education and household income. Finally, there appeared to be differences in mean resilience score by race, with non-Hispanic American Indian participants evidencing the lowest scores (13.0). 


\begin{tabular}{|c|c|c|}
\hline Variable & Mean Resilience Scores (Standard deviation) & P-value \\
\hline Gender & & $<0.001$ \\
\hline Female & $13.8(5.0)$ & \\
\hline Male & $14.4(5.1)$ & \\
\hline Age & & 0.002 \\
\hline Age $<30$ & $13.9(5.0)$ & \\
\hline Age 30 & $14.5(5.1)$ & \\
\hline Race/Ethnicity & & $<0.001$ \\
\hline Non-Hispanic White & $14.3(5.0)$ & \\
\hline Non-Hispanic Black & $14.4(5.1)$ & \\
\hline Non-Hispanic American Indian & $13.0(4.9)$ & \\
\hline Non-Hispanic Asian & $13.7(4.9)$ & \\
\hline Other Non-Hispanics & $15.0(6.0)$ & \\
\hline Hispanic & $14.4(4.9)$ & \\
\hline Highest Level of Education Achieved & & $<0.001$ \\
\hline Less than High School & $12.0(4.7)$ & \\
\hline Less than College & $14.2(5.0)$ & \\
\hline College Degree & $15.8(4.7)$ & \\
\hline More than College & $16.4(4.7)$ & \\
\hline Household Income & & $<0.001$ \\
\hline$<\$ 20,000$ & $12.6(5.2)$ & \\
\hline$\$ 20-49,999$ & $12.3(5.1)$ & \\
\hline$\$ 50-99,999$ & $13.6(4.9)$ & \\
\hline$\$ 100-149,999$ & $15.2(4.9)$ & \\
\hline$>\$ 150,000$ & $16.0(4.7)$ & \\
\hline
\end{tabular}

For the evaluation of our scale's discriminant validity, we found a significant negative correlation between AHRI scores and CESD-10 scores for depression $(r=-0.64, p<0.001)$. Table 3 and Supplementary Figure 2 both highlight that resilience scores decreased as depression scores increased. In addition, as seen in Table 5, the mean CESD-10 score for participants in the low Add Health resilience category was 11.8 and significantly different via pairwise comparisons to the mean CESD-10 scores in the medium and high Add Health resilience categories (mean CESD-10 scores 5.6 and 2.8, respectively, Bonferroni corrected pvalues $<0.001$ ). Over half of the patients in the low resilience category qualified for a diagnosis of depression per the CESD-10. Finally, there were significantly more participants who reported ever receiving a diagnosis of depression, anxiety or post-traumatic stress in the low AHRI score category than in the medium or high resilience categories (Bonferroni corrected $\mathrm{p}<0.001$ for all). 
Table 5. Summary of Discriminant Construct Validity Findings

\section{Measure}

Add Health Resilience Category

P-

values

\begin{tabular}{|c|c|c|c|c|}
\hline & $\begin{array}{c}\text { Low } \\
(\mathrm{n}=1726)\end{array}$ & $\begin{array}{c}\text { Medium } \\
(\mathrm{n}=6723)\end{array}$ & $\begin{array}{c}\text { High } \\
(\mathrm{n}=1403)\end{array}$ & \\
\hline CESD-10 Score & $\begin{array}{l}11.8(\mathrm{SD} \\
4.9)\end{array}$ & $5.6(3.8)$ & $2.8(2.6)$ & $<0.001$ \\
\hline Depressed per CESD-10 & $56.8 \%$ & $10.8 \%$ & $1.6 \%$ & $<0.001$ \\
\hline \multicolumn{5}{|l|}{ Ever received diagnosis of: } \\
\hline Depression & $33.6 \%$ & $13.3 \%$ & $6.5 \%$ & $<0.001$ \\
\hline Anxiety & $21.8 \%$ & $9.6 \%$ & $6.3 \%$ & $<0.001$ \\
\hline $\begin{array}{l}\text { Post-traumatic stress } \\
\text { disorder }\end{array}$ & $5.6 \%$ & $2.2 \%$ & $1.0 \%$ & $<0.001$ \\
\hline
\end{tabular}

\section{Discussion}

In this study, we showed that data from the National Longitudinal Study of Adolescent to Adult Health could be used to retrospectively create a global resilience scale that demonstrated appropriate psychometric properties among young adults. Using our scale, we found that women had significantly lower resilience scores than men in this cohort. This has been documented by others noting gender differences in resilience using other scales. ${ }^{12,18,23}$ We also found that older participants had slightly higher resilience scores than their younger counterparts within the same wave. This is in contrast to the findings of the original CD-RISC validation study, ${ }^{12}$ where authors found no correlation between age and CD-RISC score. It is possible that we were able to capture differences in resilience by age not previously documented as our sample size was over ten times larger than the initial validation study.

There is ongoing debate about whether resilience should be studied as a personality trait, a behavioral outcome or a dynamic process that can be modulated, which have led to a variety of ways in which resilience has been operationalized and measured. ${ }^{5}$ This is reflected in the previous work that has utilized Add Health data to explore the concept of resilience. Some research groups have written about resilience in the Add Health cohort as a description of an outcome, exploring how or why individual or contextual factors may render some participants "resilient" to a pre-specified poor outcome, but without concretely measuring resilience itself. ${ }^{35-37}$ Another angle has been the relationship between poor outcomes like depression and individual concepts that are believed to represent one aspect of resiliency, such as personal agency ${ }^{8}$, optimism ${ }^{10}$ or social support. ${ }^{11}$ In doing so, these researchers have utilized Add Health data to create a scale that operationalizes the specific resilience-related concept in question. For instance, Hitlin and Elder performed exploratory factor analysis using Wave 1 data to construct a measurement model of agency ${ }^{8}$ that has subsequently been used by others to measure the impact of agency on adolescent depression. ${ }^{10}$ 
Our work built on this concept but utilized data from when Add Health participants were firmly in their adult years (ages 24-32). In addition, it expanded existing work by creating an instrument that aims to capture a more global picture of resilience, rather than one aspect or factor related to individual resilience. The three major factors we found in the AHRI we constructed (personal competence, feeling supported vs. overwhelmed, and optimism) reflect major themes seen in prospectively validated resilience scales, ${ }^{7,12}$ which speaks to the face validity of the AHRI and situates it within what has traditionally been believed to be important when assessing individual resilience. In addition, the methodology we used to construct the AHRI highlights the ability to take existing rich cohort data and re-purpose it for the identification of important constructs and outcomes beyond those conceived of during the design of the original study.

We do acknowledge some limitations. Given the existing nature of this data and the inability to re-contact participants, we were unable to assess the consistency of responses to our Add Health resilience scale over time, such as by looking at test-retest reliability. In addition, although Add Health has contacted the same participants over several waves, not every wave of interviews asks the same items and many of the resilience-related questions changed with each wave of interviews. Thus, participants' responses to the items we used to construct our resilience scale could not be assessed at any other time point using existing data.

\section{Conclusions}

The Add Health Resilience Instrument (AHRI) derived via interview data from a nationally representative longitudinal cohort study showed that a resilience scale could be constructed retrospectively and evidence good psychometric properties. This scale can be applied in future studies that utilize Add Health data to further explore the relationship between resilience, health, health behaviors and community context in young adults.

\section{List Of Abbreviations}

Add Health: Adolescent to Adult Health

AHRI: Add Health Resilience Instrument

CD-RISC: Connor-Davidson Resilience Scale

CESD-10: Centers for Epidemiologic Studies Depression Scale-10

\section{Declarations}

Ethics approval and consent to participate: This study was deemed exempt by our local institutional review board to the publicly available de-identified nature of the Add Health data. 
Availability of data and material: Parts of the datasets generated and/or analysed during the current study are publically available in the Adolescent to Adult Health repository (https://www.cpc.unc.edu/projects/addhealth/documentation/publicdata). However this study utilized the extensive restricted-use data available by contractual agreement.

Competing interests: The authors declare that they have no competing interests

Funding: This research uses data from Add Health, a program project directed by Kathleen Mullan Harris and designed by J. Richard Udry, Peter S. Bearman, and Kathleen Mullan Harris at the University of North Carolina at Chapel Hill, and funded by grant P01-HD31921 from the Eunice Kennedy Shriver National Institute of Child Health and Human Development, with cooperative funding from 23 other federal agencies and foundations. Special acknowledgment is due Ronald R. Rindfuss and Barbara Entwisle for assistance in the original design. Information on how to obtain the Add Health data files is available on the Add Health website (http://www.cpc.unc.edu/addhealth). No direct support was received from grant P01-HD31921 for this analysis.

Authors' contributions: DMW conceptualized of the study, analyzed and interpreted the data and wrote the manuscript. MP assisted with dataset creation and statistical analysis. SAL oversaw the study, assisted with analysis and interpretation and provided edits. All authors read and approved the final manuscript

Acknowledgements: We would like to thank Ms. Ali Chandler for her assistance in editing and preparing this manuscript.

\section{Authors' information (optional): N/A}

\section{References}

1. Luthar SS, Cicchetti D, Becker B. The construct of resilience: a critical evaluation and guidelines for future work. Child Dev. 2000;71(3):543-562.

2. Solivan AE, Wallace ME, Kaplan KC, Harville EW. Use of a resiliency framework to examine pregnancy and birth outcomes among adolescents: A qualitative study. Fam Syst Health. 2015;33(4):349-355.

3. Mittelmark MB, Bull T. The salutogenic model of health in health promotion research. Glob Health Promot. 2013;20(2):30-38.

4. MacLeod S, Musich S, Hawkins K, Alsgaard K, Wicker ER. The impact of resilience among older adults. Geriatr Nurs. 2016;37(4):266-272.

5. Hu T, Zhang D, Wang J. A meta-analysis of the trait resilience and mental health. Pers Individ Dif. 2015;76:18-27.

6. Windle G, Bennett KM, Noyes J. A methodological review of resilience measurement scales. Health Qual Life Outcomes. 2011;9:8. 
7. Smith BW, Dalen J, Wiggins K, Tooley E, Christopher P, Bernard J. The brief resilience scale: assessing the ability to bounce back. Int J Behav Med. 2008;15(3):194-200.

8. Hitlin S, Elder GH. Agency: An Empirical Model of an Abstract Concept. Adv Life Course Res. 2006;11:33-67.

9. Harris, K.M., C.T. Halpern, E. Whitsel, J. Hussey, J. Tabor, P. Entzel, and J.R. Udry. The National Longitudinal Study of Adolescent to Adult Health: Research Design. http://www.cpc.unc.edu/projects/addhealth/design. Published 2009. Accessed March 4, 2019.

10. Williams AL, Merten MJ. Linking community, parenting, and depressive symptom trajectories: testing resilience models of adolescent agency based on race/ethnicity and gender. J Youth Adolesc. 2014;43(9):1563-1575.

11. Wickrama KAS, Bryant CM. Community Context of Social Resources and Adolescent Mental Health. J Marriage and Family. 2003;65(4):850-866.

12. Connor KM, Davidson JRT. Development of a new resilience scale: the Connor-Davidson Resilience Scale (CD-RISC). Depress Anxiety. 2003;18(2):76-82.

13. Green KT, Hayward LC, Williams AM, et al. Examining the factor structure of the Connor-Davidson Resilience Scale (CD-RISC) in a post-9/11 U.S. military veteran sample. Assessment. 2014;21(4):443451.

14. Solano JPC, Bracher ESB, Faisal-Cury A, et al. Factor structure and psychometric properties of the Connor-Davidson resilience scale among Brazilian adult patients. Sao Paulo Med J. May 2016. doi:10.1590/1516-3180.2015.02290512

15. Fu C, Leoutsakos J-M, Underwood C. An examination of resilience cross-culturally in child and adolescent survivors of the 2008 China earthquake using the Connor-Davidson Resilience Scale (CD-RISC). J Affect Disord. 2014;155:149-153.

16. Campbell-Sills L, Stein MB. Psychometric analysis and refinement of the Connor-davidson Resilience Scale (CD-RISC): Validation of a 10-item measure of resilience. J Trauma Stress. 2007;20(6):10191028.

17. Blanco V, Guisande MA, Sánchez MT, Otero P, Vázquez FL. Spanish validation of the 10-item ConnorDavidson Resilience Scale (CD-RISC 10) with non-professional caregivers. Aging Ment Health. November 2017:1-6.

18. Notario-Pacheco B, Solera-Martínez M, Serrano-Parra MD, Bartolomé-Gutiérrez R, García-Campayo J, Martínez-Vizcaíno V. Reliability and validity of the Spanish version of the 10-item Connor-Davidson Resilience Scale (10-item CD-RISC) in young adults. Health Qual Life Outcomes. 2011;9:63.

19. Terwee CB, Bot SDM, de Boer MR, et al. Quality criteria were proposed for measurement properties of health status questionnaires. J Clin Epidemiol. 2007;60(1):34-42.

20. Kane R. Outcome Measures. Gaithersburg: Aspen Publishers; 1997.

21. Streiner DL, Norman GR, Cairney J. Health Measurement Scales: A Practical Guide to Their Development and Use. Oxford University Press; 2015. 
22. BrckaLorenz A, Chiang Y, Nelson Laird T. Internal Consistency. FSSE Psychometric Portfolio. 2013. http://fsse.indiana.edu/pdf/pp/2013/FSSE13_Internal_Consistency_Reliability.pdf. Accessed November 30, 2018.

23. Rodríguez-Rey R, Alonso-Tapia J, Hernansaiz-Garrido H. Reliability and validity of the Brief Resilience Scale (BRS) Spanish Version. Psychol Assess. 2016;28(5):e101-e110.

24. Lee J, Blackmon BJ, Cochran DM, Kar B, Rehner TA, Gunnell MS. Community Resilience, Psychological Resilience, and Depressive Symptoms: An Examination of the Mississippi Gulf Coast 10 Years After Hurricane Katrina and 5 Years After the Deepwater Horizon Oil Spill. Disaster Med Public Health Prep. 2018;12(2):241-248.

25. Min J-A, Lee C-U, Chae J-H. Resilience moderates the risk of depression and anxiety symptoms on suicidal ideation in patients with depression and/or anxiety disorders. Compr Psychiatry. 2015;56:103-111.

26. Thompson NJ, Fiorillo D, Rothbaum BO, Ressler KJ, Michopoulos V. Coping strategies as mediators in relation to resilience and posttraumatic stress disorder. J Affect Disord. 2018;225:153-159.

27. Eaton WW, Muntaner C, Smith C, Tien A, Ybarra M. Center for Epidemiologic Studies Depression Scale: Review and revision (CESD and CESD-R). In: Maruish ME, ed. The Use of Psychological Testing for Treatment Planning and Outcomes Assessment. Mahwah, New Jersey: Lawrence Erlbaum; 2004:363-377.

28. Crockett LJ, Randall BA, Shen Y-L, Russell ST, Driscoll AK. Measurement equivalence of the center for epidemiological studies depression scale for Latino and Anglo adolescents: a national study. $J$ Consult Clin Psychol. 2005;73(1):47-58.

29. Roberts RE, Lewinsohn PM, Seeley JR. Screening for adolescent depression: a comparison of depression scales. J Am Acad Child Adolesc Psychiatry. 1991;30(1):58-66.

30. Andresen EM, Malmgren JA, Carter WB, Patrick DL. Screening for Depression in Well Older Adults: Evaluation of a Short Form of the CES-D. Am J Prev Med. 1994;10(2):77-84.

31. Primack BA, Swanier B, Georgiopoulos AM, Land SR, Fine MJ. Association between media use in adolescence and depression in young adulthood: a longitudinal study. Arch Gen Psychiatry. 2009;66(2):181-188.

32. Barnert ES, Abrams LS, Dudovitz R, et al. What Is the Relationship Between Incarceration of Children and Adult Health Outcomes? Acad Pediatr. June 2018. doi:10.1016/j.acap.2018.06.005

33. Suglia SF, Demmer RT, Wahi R, Keyes KM, Koenen KC. Depressive Symptoms During Adolescence and Young Adulthood and the Development of Type 2 Diabetes Mellitus. Am J Epidemiol. 2016;183(4):269-276.

34. Comparing 2008 American Community Survey Data. United States Census Bureau. https://www.census.gov/programs-surveys/acs/guidance/comparing-acs-data/2008.html. Published June 17, 2008. Accessed March 15, 2019.

35. Newsome J, Sullivan CJ. Resilience and vulnerability in adolescents: genetic influences on differential response to risk for delinquency. J Youth Adolesc. 2014;43(7):1080-1095. 
36. Langenkamp AG. Academic Vulnerability and Resilience during the Transition to High School: The Role of Social Relationships and District Context. Sociol Educ. 2010;83(1):1-19.

37. Brody GH, Yu T, Miller GE, Chen E. Resilience in Adolescence, Health, and Psychosocial Outcomes. Pediatrics. 2016;138(6). doi:10.1542/peds.2016-1042

\section{Supplementary Files}

This is a list of supplementary files associated with this preprint. Click to download.

- supplement1.pdf 